\title{
Depletions of Elements from the Gas Phase: A Guide on Dust Compositions
}

\author{
Edward B. Jenkins* \\ Princeton University Observatory \\ E-mail: ebjeastro.princeton.edu
}

\begin{abstract}
Ultraviolet spectra of stars recorded by orbiting observatories since the 1970's have revealed absorption features produced by atoms in their favored ionization stages in the neutral ISM of our Galaxy. Most elements show abundances relative to hydrogen that are below their values in stars, indicating their removal by condensation into solid form. The relative amounts of these depletions vary from one location to the next, and different elements show varying degrees of depletion. In a study of abundances along 243 different sight lines reported in more than 100 papers, Jenkins (2009) characterized the systematic patterns for the depletions of 17 different elements, and these results in turn were used to help us understand the compositions of dust grains. Since the conclusions are based on differential depletions along different sightlines, they are insensitive to errors in the adopted values for the total element abundances. Some of the more remarkable conclusions to emerge from this study are that (1) oxygen depletions in the denser gas regions (but not as dense as the interiors of molecular clouds) are stronger than what we can expect from just the formation of silicates and metallic oxides, and (2) the chemically inert element krypton shows some evidence for weak depletion, perhaps as a result of trapping within water clathrates or binding with $\mathrm{H}_{3}^{+}$.
\end{abstract}

The Life Cycle of Dust in the Universe: Observations, Theory, and Laboratory Experiments 18-22 November, 2013

Taipei, Taiwan

\footnotetext{
* Speaker.
} 


\section{Background}

When various elements condense into solid form in the interstellar medium, there are profound reductions in the relative abundances of many types of heavy atoms and ions in the gas phase. We can obtain insights on the compositions of the dust grains by studying the strengths of depletions of different gas constituents below what we believe to be their intrinsic abundances. Virtually all of the free atoms in the neutral interstellar medium are in their ground electronic states.

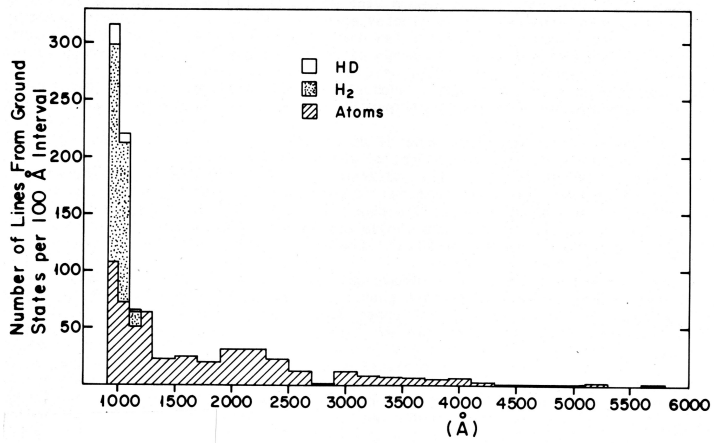

Figure 1: Line density vs. wavelength For nearly all of the important atomic species, the energy separations of excited levels above the ground state are larger than the energies of transitions in the visible part of the spectrum. Hence, as shown in Fig. 1, for absorption spectroscopy a vast majority of the useful features are situated in the UV part of the spectrum; this fact was recognized long ago by L. Spitzer \& Zabriskie [1]. For studying gas in our Galaxy or nearby systems, these transitions can only be accessed by observatories in space. Except for the elements Be [2-4] and Ti [5-12] and some comparisons of the abundances of $\mathrm{Ca}$ to $\mathrm{Na}$ with velocity [13-16], research on interstellar atomic abundances relied almost entirely on recording stellar spectra over the range extending from the Lyman limit $(912 \AA)$ up to about $3000 \AA$, where the Earth's atmosphere becomes transparent.

The Copernicus satellite [17], launched in 1972, was the first major observatory that had the resolution and sensitivity for productive research on atomic abundances in the interstellar medium $[18,19]$. The earliest results from this facility [20] exhibited a pattern of element depletions that led Field [21] to conclude that the severities of depletions are strongly linked to the temperatures at which the respective elements condense into solid compounds in matter being expelled from the outer portions of stellar atmospheres. This formation mechanism, along with the creation of dust in supernova ejecta and the further growth of grains in dense gas complexes in space, still remains a central theme in theories of dust production. A decade of observations with Copernicus reached a climax with the study of element abundances across a broad range of sight lines toward bright stars in our Galaxy [22], revealing that the strengths of depletions were strongly linked to the average gas densities, indicating that the growth and destruction of grains depend on local conditions.

Following the era of Copernicus, major advances in achieving higher accuracy and viewing fainter stars arose from observations by the Goddard High Resolution Spectrograph [23] aboard the Hubble Space Telescope (HST) soon after its launch in 1990 [24-30]. Savage \& Sembach [31] provided an excellent review of these early accomplishments with HST. A major upgrade in the ability to record a broad wavelength coverage at high resolution was provided when the Space Telescope Imaging Spectrograph was installed on the second servicing mission of HST in 1997 [32]. The Far Ultraviolet Spectroscopic Explorer (FUSE), launched in 1999, extended our coverage to a wavelength band between the Lyman limit and the short-wavelength cutoff of HST. This facility allowed us to determine the amount of $\mathrm{H}_{2}$ that accompanied the $\mathrm{H} \mathrm{I}$ gas and also gave us an access to some important atomic transitions that were not available to HST [33]. 


\section{A Unified Representation of Depletions}

During the first 35 years of observations using the orbiting observatories to gather absorption line data, there had emerged a large number of publications that reported on a heterogeneous mix of different element abundances over a diverse collection of sight lines. The results by themselves were of significant value and led to interesting conclusions. Nevertheless, there still remained a need to consolidate the information from different sources and then systematically describe the outcomes in terms that linked the depletion of each element to those of the others, and at the same time, accounted for the variations that were known to take place from one region to the next. This task was accomplished in 2009 by Jenkins [34], who interpreted the abundances of 17 different elements reported in more than 100 papers that covered 243 sight lines to different stars. He developed a simple unification scheme that described in straightforward parametric forms the logarithmic depletion factor of any element $X$ from the gas phase,

$$
\left[X_{\text {gas }} / \mathrm{H}\right]=\log \left(\frac{N(X)}{N(\mathrm{H})}\right)_{\text {obs }}-\log \left(\frac{X}{\mathrm{H}}\right)_{\text {ref }},
$$

where $N(X)$ is the column density of element $X, N(\mathrm{H})$ represents the column density of hydrogen in both atomic and molecular form, i.e., $N(\mathrm{H} \mathrm{I})+2 N\left(\mathrm{H}_{2}\right)$, and the reference abundance ratio $(X / \mathrm{H})_{\text {ref }}$ can be taken from either the abundances measured for $\mathrm{B}$ type stars or the Sun. It follows that the missing atoms that make up the relative contribution of element $X$ in the form of dust grains or large molecules is given (in linear terms) by the expression

$$
\left(X_{\text {dust }} / \mathrm{H}\right)=(X / \mathrm{H})_{\text {ref }}\left(1-10^{\left[X_{\text {gas }} / \mathrm{H}\right]}\right) .
$$

Aside from constructing a unification scheme for element depletions that could lead to dust compositions, Jenkins had to exercise many quality control procedures and devise corrections to the earlier results that accounted for updated values for the transition strengths.

The basic foundation for the construction of a unified representation of depletions is based on the observation that any particular combination of two elements exhibits logarithmic depletions that have a linear relationship with each other. This concept is illustrated in Fig. 2. When compared to the logarithms of the depletions of $\mathrm{P}$, the logarithmic depletions of $\mathrm{Mg}$ and $\mathrm{Fe}$ show a linear trend. Different sight lines have varying degrees of depletions of all three elements, and the overall severity of such depletions for any sight line can be characterized by a single parameter, which Jenkins designated as $F_{*}$. The differences in the slopes and intercepts of the trend 
lines representing a progression of $F_{*}$ can be represented by just two additional parameters that are unique to each element. The $F_{*}$ sight-line parameter depends only on observed depletions, and it replaces the average density $\langle n(\mathrm{H})\rangle \equiv\left[N(\mathrm{H} \mathrm{I})+2 N\left(\mathrm{H}_{2}\right)\right] / d$ or molecular hydrogen fraction $f\left(\mathrm{H}_{2}\right) \equiv 2 N\left(\mathrm{H}_{2}\right) /\left[N(\mathrm{H} \mathrm{I})+2 N\left(\mathrm{H}_{2}\right)\right]$ as a discriminant that had been used by earlier investigators [22, 30, 35]. The adopted scale for $F_{*}$ was arbitrary: Jenkins defined $F_{*}=0.0$ to correspond to the weakest depletions that appeared in the survey, while $F_{*}=1.0$ represented the depletions seen toward the well studied, strongly depleted $-15 \mathrm{~km} \mathrm{~s}^{-1}$ component toward the star $\zeta$ Oph [27]. Occasionally, sight lines exhibited values of $F_{*}$ outside the range $0.0-1.0$.

Jenkins characterized the depletion trends for different elements using the equation

$$
\left[X_{\mathrm{gas}} / \mathrm{H}\right]=B_{X}+A_{X}\left(F_{*}-z_{X}\right)
$$

Fig. 3 illustrates the meanings of the parameters $A_{X}, B_{X}$, and $z_{X}$, and Table 1 lists their values that Jenkins derived for the 17 elements in his study, along with the adopted reference abundances (taken to be the solar abundances $(X / H) \odot$ specified by Lodders [36]). One drawback of having to rely on reference abundances is the fact that some of them are controversial. Figure 4 shows that there are some disagreements between B-star abundances [37] and solar abundances [38]. We can ask the question "Why is it that for many heavy elements the abundances in the Sun (4.5 Gyr old) appear to higher than those in young B stars (several tens of Myr old)?" The answer may arise from a suggestion by Lyubimkov [39] that ionizations in the standard stellar atmosphere models for early-type stars are underestimated. From Eq. 2.2 it should be clear that the answers that we will obtain for the dust elemental compositions will depend on the adopted reference abundances.

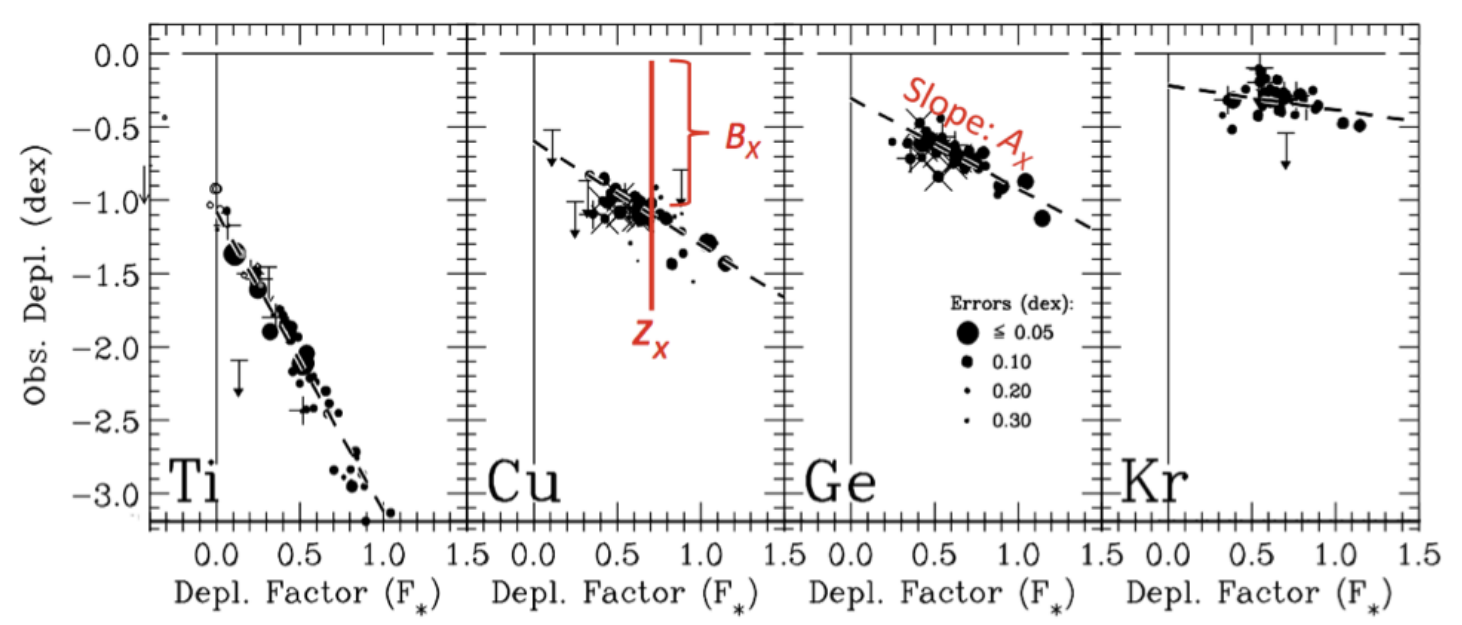

Figure 3: Examples for 4 different elements that illustrate the linear equation (Eq. 2.3) that relates the logarithmic depletion factor for an element $X$ to the sight-line parameter $F_{*}$. The zero point $z_{X}$ for $F_{*}$ is chosen such that, with the observations and their errors at hand, the covariance of the errors in the other two parameters, $A_{X}$ (slope) and $B_{X}$ (vertical offset) is zero. 


\begin{tabular}{lcccc}
\hline Elem. & $\begin{array}{c}\text { Adopted } \\
X\end{array}$ & $A_{X}$ & $B_{X}$ & $z_{X}$ \\
\hline $\mathrm{C}$ & $8.46 \pm 0.04$ & $-0.101 \pm 0.229$ & $-0.193 \pm 0.060$ & 0.803 \\
$\mathrm{~N}$ & $7.90 \pm 0.11$ & $-0.000 \pm 0.079$ & $-0.109 \pm 0.111$ & 0.550 \\
$\mathrm{O}$ & $8.76 \pm 0.05$ & $-0.225 \pm 0.053$ & $-0.145 \pm 0.051$ & 0.598 \\
$\mathrm{Mg}$ & $7.62 \pm 0.02$ & $-0.997 \pm 0.039$ & $-0.800 \pm 0.022$ & 0.531 \\
$\mathrm{Si}$ & $7.61 \pm 0.02$ & $-1.136 \pm 0.062$ & $-0.570 \pm 0.029$ & 0.305 \\
$\mathrm{P}$ & $5.54 \pm 0.04$ & $-0.945 \pm 0.051$ & $-0.166 \pm 0.042$ & 0.488 \\
$\mathrm{Cl}$ & $5.33 \pm 0.06$ & $-1.242 \pm 0.129$ & $-0.314 \pm 0.065$ & 0.609 \\
$\mathrm{Ti}$ & $5.00 \pm 0.03$ & $-2.048 \pm 0.062$ & $-1.957 \pm 0.033$ & 0.430 \\
$\mathrm{Cr}$ & $5.72 \pm 0.05$ & $-1.447 \pm 0.064$ & $-1.508 \pm 0.055$ & 0.470 \\
$\mathrm{Mn}$ & $5.58 \pm 0.03$ & $-0.857 \pm 0.041$ & $-1.354 \pm 0.032$ & 0.520 \\
$\mathrm{Fe}$ & $7.54 \pm 0.03$ & $-1.285 \pm 0.044$ & $-1.513 \pm 0.033$ & 0.437 \\
$\mathrm{Ni}$ & $6.29 \pm 0.03$ & $-1.490 \pm 0.062$ & $-1.829 \pm 0.035$ & 0.599 \\
$\mathrm{Cu}$ & $4.34 \pm 0.06$ & $-0.710 \pm 0.088$ & $-1.102 \pm 0.063$ & 0.711 \\
$\mathrm{Zn}$ & $4.70 \pm 0.04$ & $-0.610 \pm 0.066$ & $-0.279 \pm 0.045$ & 0.555 \\
$\mathrm{Ge}$ & $3.70 \pm 0.05$ & $-0.615 \pm 0.083$ & $-0.725 \pm 0.054$ & 0.690 \\
$\mathrm{Kr}$ & $3.36 \pm 0.08$ & $-0.166 \pm 0.103$ & $-0.332 \pm 0.083$ & 0.684 \\
\hline
\end{tabular}

Table 1: The depletion parameters for 17 elements.

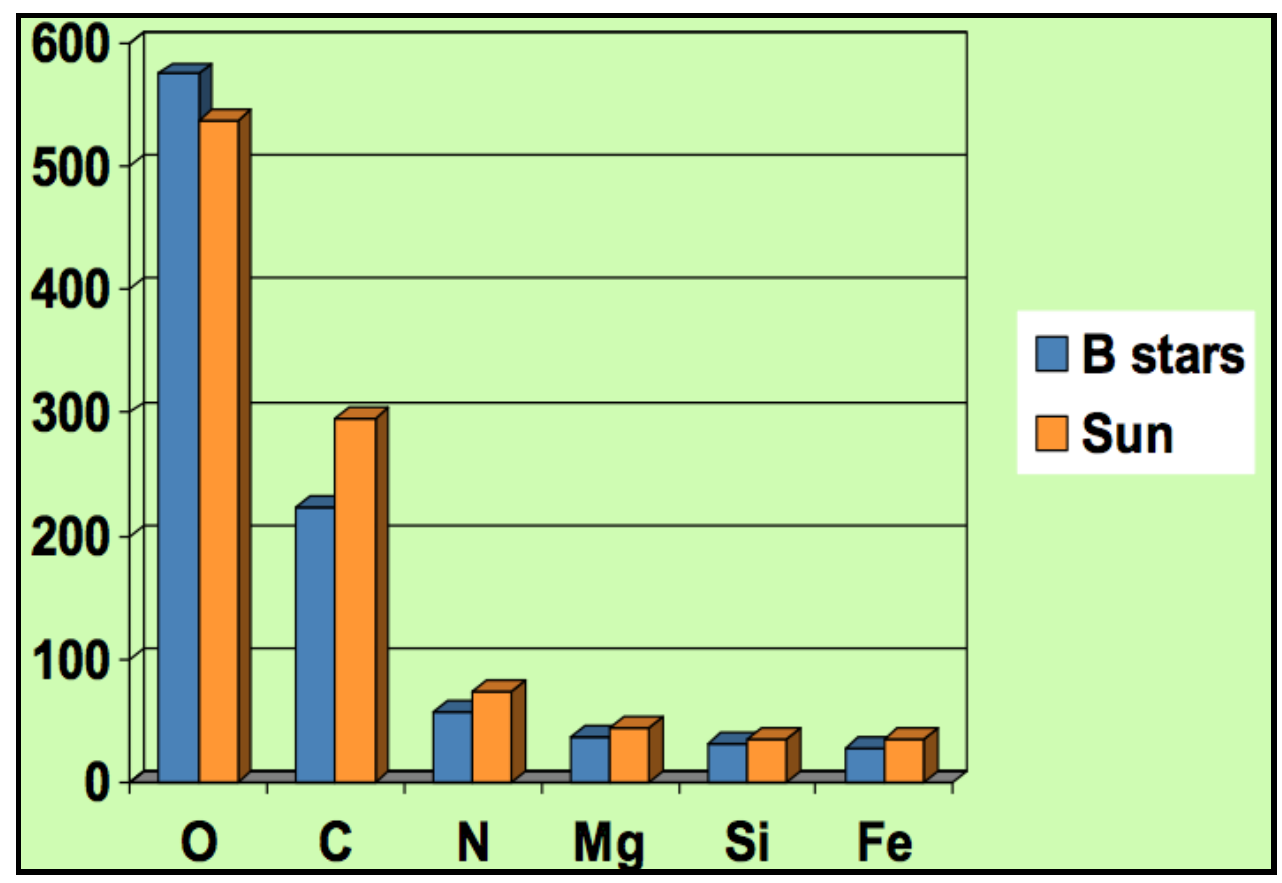

Figure 4: A comparison of B-star [37] and solar [38] reference abundances for some selected elements. The units on the $y$-axis correspond to parts per million $\mathrm{H}$ atoms. 


\section{The Buildup of Dust Grains}

A method for overcoming the effects arising from uncertainties in the reference abundances is to develop an interpretation that avoids having to use them in the first place. Instead of attempting to derive the absolute quantities of elements sequestered in the grains, we can change our objective to that of monitoring the changes in element abundances as we progress from regions with low values of $F_{*}$ to ones where the grains have advanced to much greater stages of growth, as indicated by larger values of $F_{*}$. If we substitute the right-hand side of Eq. 2.3 into Eq. 2.2 and differentiate the result with respect to $F_{*}$, we find that

$$
\begin{aligned}
d\left(X_{\text {dust }} / \mathrm{H}\right) / d F_{*} & =-(\ln 10)(X / \mathrm{H})_{\odot} A_{X} 10^{B_{X}+A_{X}\left(F_{*}-z_{X}\right)} \\
& =-(\ln 10) A_{X}\left(X_{\text {gas }} / \mathrm{H}\right)_{F_{*}}
\end{aligned}
$$

We now have an expression that makes use of only the slope $A_{X}$ and the (linear) expression for the relative abundance of the element $X$ with respect to hydrogen in the gas for any particular value of $F_{*}$. The resulting consumption rates for different elements are shown in Fig. 5. These rates change as the grains mature, indicating that the compositions are driven by the abundance of the remaining gaseous feedstocks (free atoms) and perhaps also by the nature of the existing compounds in the grains with which new atoms can combine. For instance, we can see from the differences in the bar lengths in the figure that there is little change in the consumption rates of $\mathrm{C}$ and $\mathrm{O}$, presumably because they remain abundant even after many grains have formed, whereas a less abundant element that starts out being rapidly depleted, such as Ti, arrives at the point where there are few atoms left to contribute to further grain growth. From the evidence that is presented here, it should be clear that the compositions of the outer mantles of grains differ from their inner cores that formed from gases that had higher concentrations of heavy atoms.

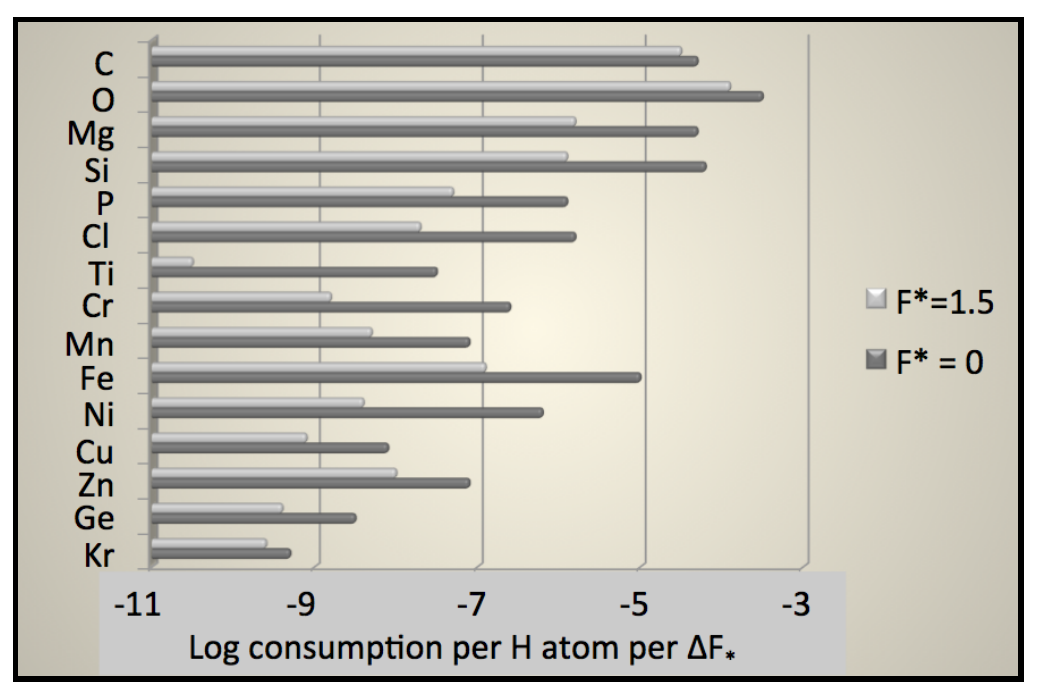

Figure 5: Logarithms of the element consumption rates $d\left(X_{\text {dust }} / \mathrm{H}\right) / d F_{*}$, as defined by Eq. 3.1, for two values of $F_{*}$. The elements here are the same as those listed in Table 1, except for nitrogen, which exhibits no measurable changes in depletion as a function of $F_{*}$ (i.e., $A_{N}=0.00$ ). 


\section{Properties of the Sight Lines}

In $\S 2$ we considered that the basic premise for the development of the $F_{*}$ parameter was that the collective depletion of many elements gives the most reliable indication of the overall level of grain production for any given sight line, after one applies proper scalings to account for the elements' depletion behaviors. The small scatter in the relationships between the depletions of most elements and $F_{*}$ justifies this choice, which appears to be more satisfactory the more traditional methods of using the environmental factors $\langle n(\mathrm{H})\rangle$ or $f\left(\mathrm{H}_{2}\right)$ as guides for grain development. Nevertheless, it is of interest to go back and see how well those measures compare with determinations of $F_{*}$. Figure 6 shows these relationships. It appears that $\langle n(\mathrm{H})\rangle$ is a better predictor for $F_{*}$ than $f\left(\mathrm{H}_{2}\right)$. This figure also shows that distances $|z|$ above or below the Galactic plane seem to have no systematic effects on the results.
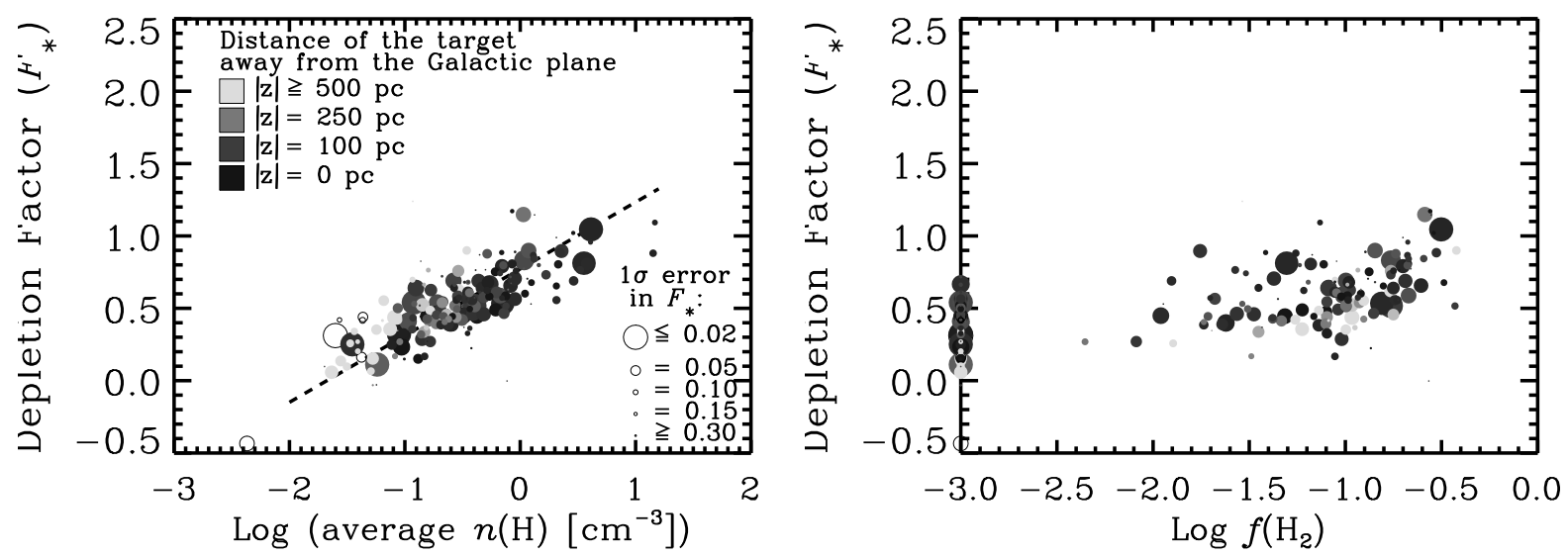

Figure 6: Left-hand panel: The trend of $F_{*}$ as a function of the logarithm of the average density along the respective sight lines $\langle n(\mathrm{H})\rangle$. As indicated by the legends, the sizes of the circles indicate the errors in $F_{*}$, and their shades of gray indicate the distances of the stars from the Galactic plane. Open circles indicate cases where $\log N(\mathrm{H})<19.5$, where ionization effects may play a role in distorting the results. The leastsquares best fit to the trend is indicated by the dashed line. Right-hand panel: The trend of $F_{*}$ against the logarithm of the fraction of hydrogen in molecular form $f\left(\mathrm{H}_{2}\right)$. The sizes and gray levels of the points are the same as in the left-hand panel. Cases where $\log f\left(\mathrm{H}_{2}\right)<-3.0$ are all grouped together on the $y$ axis of the plot.

\section{Comparisons with Condensation Temperatures}

Another issue that we can revisit is how well do the element depletion factors scale with the condensation temperature $T_{c}$. This quantity has a specific meaning related to the formation of solid compounds when a cosmic mixture of free atoms experiences a progressive decrease in temperature at some specific pressure, as in the expanding stellar atmospheres or nebulae considered by Field [21]. There are, however, alternative processes that can be important. For instance, we can recognize that $T_{c}$ correlates well with other properties of atoms, such as ionization potentials [40], binding energies to PAHs [41], or sublimation energies [42] that govern the resistance of compounds to sputtering that can ultimately lead to the destruction of grain materials and the return of 
atoms to the gas phase. Over any large selection of elements, these properties are correlated with each other (e.g. see Fig. 5 of Ramírez, et al. [43] for a plot of $T_{c}$ vs. first ionization potentials), so identifying the most important processes is not straightforward. Nevertheless, we can propose that $T_{c}$ is a useful parameter to examine, even if it does not represent faithfully the most important formation and destruction routes.

The left-hand panel of Figure 7 shows how well the depletions at $F_{*}=0$ correlate with $T_{c}$. While a trend seems evident, there are some awkward cases that involve 3 elements where negative depletions are apparent (i.e., abundances that seem to be greater than their respective reference abundances). An explanation for these anomalies may rest with either bad measurements, incorrect transition $f$-values, or inaccurate reference abundances. A more satisfactory trend is seen when $A_{X}$ (the slope of logarithmic depletions vs. $F_{*}$ ) is compared with $T_{c}$. Here, the dependences on $f$-values or the adopted reference abundances are absent.
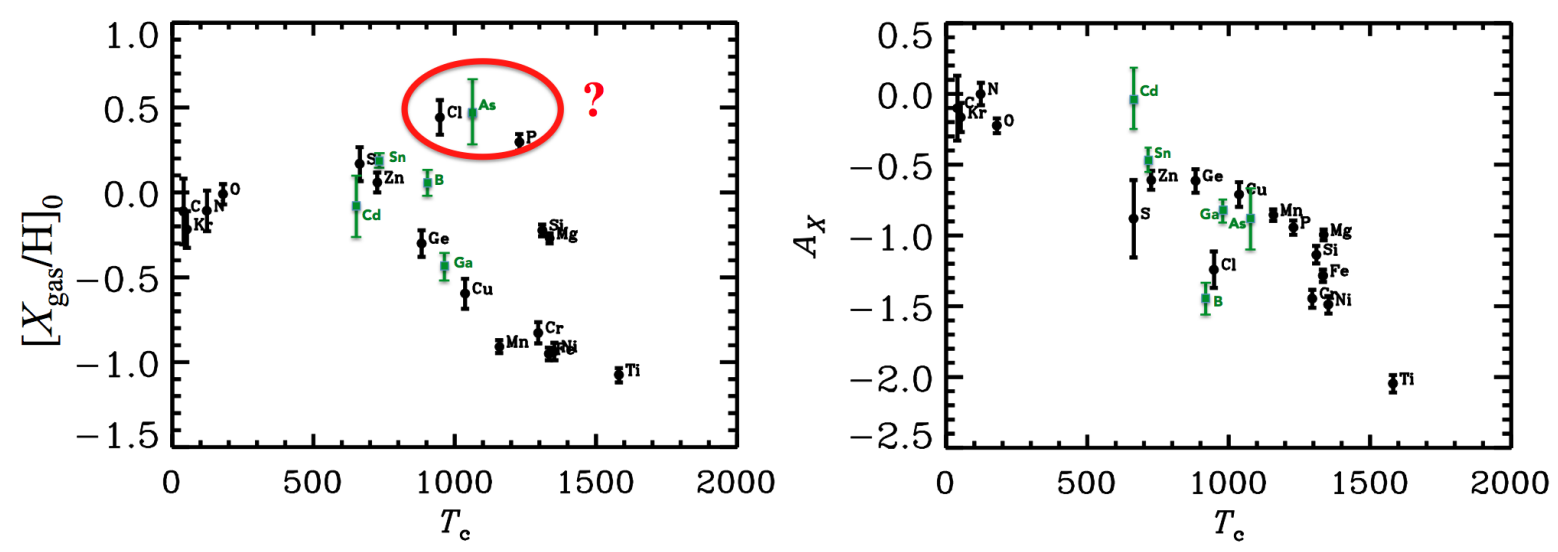

Figure 7: Left-hand panel: The trend of element depletions for sight lines having $F_{*} \approx 0$ as a function of their respective condensation temperatures $T_{c}$. The 3 elements within the red ellipse appear to exhibit interstellar abundances that are greater than the reference abundances. Right-hand panel: The dependence of $A_{X}$, the slope of logarithmic depletions vs. $F_{*}$, on $T_{c}$. In both panels, the elements shown in green are from a study of depletions by A. M. Ritchey (private communication).

Recent studies that compare the differences of abundances in the atmosphere of the Sun compared to those of solar twins and solar analogs $[44,45]^{1}$ show a pattern very similar to the $A_{X}$ vs. $T_{c}$ trend shown in Fig. 7, but much smaller in amplitude. A popular conjecture is that the refractory elements condense out to form dusty material that eventually goes into rocky planets or the interiors of gas giants, and that the remaining gas is dumped into a thin convection zone of the star. However, Ramírez, Meléndez \& Asplund [46] surveyed the differential abundances of many solar-type stars and were not able to find a good correlation of these depletions with the presence or absence of planets; the picture may be muddled by how deep the stars' convection zones were at the time when the separations took place and the planetary disks formed.

\footnotetext{
${ }^{1}$ Ramírez, et al. [44] define a solar twin as a star that has $T_{\text {eff }}$ within $100 \mathrm{~K}, \log g$ within $0.1 \mathrm{dex}$, and [Fe/H] within 0.1 dex of the respective values of the Sun. Solar analogs are main-sequence stars that have spectral types G0-G5 [45].
} 


\section{Comments about Specific Elements}

A number of interesting conclusions emerge from the findings for specific elements, as summarized in the subsections that follow.

\subsection{Carbon}

Many investigators [47-52] have expressed concerns that the $\mathrm{C}$ depletion results indicated that there was an insufficient amount of carbon in solid form to reconcile the observed optical and nearUV extinctions with calculations of the optical properties of dust grains, where the requirements for the fractional amounts of $\mathrm{C}$ in solids ranged from $150-200$ parts per million $\mathrm{H}$ atoms (ppm) [48] to 300 ppm [53]. This has often been referred to as the "carbon crisis." If we apply Eq. 2.2 to the parameters shown in Table 1, we find that the amount of $\mathrm{C}$ locked up in dust grains ranges from $\left(\mathrm{C}_{\text {dust }} / \mathrm{H}\right)=65 \mathrm{ppm}$ for $F_{*}=0.0$ to $111 \mathrm{ppm}$ for $F_{*}=1.0$. From this, one might conclude that the carbon crisis still remains. However, Sofia, et al. [54] have measured the damping wings of the $1334 \AA$ allowed transition of C II in some spectra instead of the customarily-measured strengths of the very weak intersystem line at $2325 \AA$. They obtained interstellar carbon column densities that were about 0.43 times as large as those obtained from the weak line. If the revision suggested by Sofia, et al. is correct, then the carbon crisis may be mostly resolved, since now $\left(\mathrm{C}_{\text {dust }} / \mathrm{H}\right)=$ $(192,212) \mathrm{ppm}$ for the two values of $F_{*}$. It is generally true that the $f$-values that are calculated for semi-forbidden lines are much less reliable than those for allowed transitions, which may indicate that the new $\mathrm{C}$ abundances are more trustworthy. However, an opposing consideration is the fact that Sofia, et al. had to measure damping wings on top of strong stellar C II lines, which they had to estimate from calculations of stellar model atmospheres and radiative transfer.

\subsection{Nitrogen}

Of all 17 elements studied by Jenkins [34], nitrogen appears to be only one that shows no measurable change in depletion as $F_{*}$ is increased. The entries for nitrogen in Table 1 indicates that $\left[\mathrm{N}_{\text {gas }} / \mathrm{H}\right]$ appears to hold at a steady value of $-0.109 \pm 0.111 \mathrm{dex}$, which is possibly consistent with no depletion at all. Gail \& Sedlmayr [55] suggested that the condensation of $\mathrm{N}$ into any sort of solid compound might be inhibited by the production of $\mathrm{N}_{2}$ which has a saturated bond with a very high activation energy for any gas-phase reactions that could form other molecules. This may be an attractive theoretical consideration, but the observed abundance of $\mathrm{N}_{2}$ in the interstellar medium is small $[56,57]$.

\subsection{Oxygen}

A conventional view is that oxygen in dust grains appears in the form of refractory compounds such as silicates and metallic oxides. In this category, the elements $\mathrm{Mg}, \mathrm{Si}$, and $\mathrm{Fe}$ are the only ones with significantly high abundances to be relevant. We can examine whether or not the differential depletions $\mathrm{O}$ with respect to these three elements is consistent with this picture (again, this allows us to sidestep the uncertainties in the reference abundance of $\mathrm{O}$, which, through the years, has shown large changes from one determination to the next; see $\$ 3.4$ of ref [38] for details). At $F_{*}=0.0$, Eq. 3.1 and the parameters in Table 1 tell us that $d\left(\mathrm{O}_{\text {dust }} / \mathrm{H}\right) / d F_{*}=1.6 d\left(\mathrm{Mg}+\mathrm{Si}+\mathrm{Fe}_{\text {dust }} / \mathrm{H}\right) / d F_{*}$, which is consistent with the most oxygen-rich silicate, $\mathrm{MgSiO}_{3}$ where the ratio of $\mathrm{O}$ to the other 
atoms is 1.5 . However, when $F_{*}=1.0$ the differential consumption of $\mathrm{O}$ relative to the other three elements is ten times higher! Even when we consider the $-2 \sigma$ uncertainty in $d\left(\mathrm{O}_{\text {dust }} / \mathrm{H}\right) / d F_{*}$, we find that the ratio only drops to 6 times the consumption rate of $\mathrm{Mg}+\mathrm{Si}+\mathrm{Fe}$.

Clearly, in addition to forming silicates or metallic oxides, oxygen must be in bound form with some other abundant element. We can rule out oxides of nitrogen, because the differential depletion of this element is about zero (see \$6.2). Water ice seems to be an attractive prospect; it is known to be abundant in very dense clouds and protostellar nebulae [58], but various surveys of the $3.6 \mu \mathrm{m}$ ice band shows a linear trend with visual extinction $A_{V}$ that extrapolates to zero for $A_{V}=2.6-5$ [59-61] (most $A_{V}$ values for the sight lines in the UV surveys are less than 1). The molecules $\mathrm{CO}, \mathrm{CO}_{2}$ and $\mathrm{O}_{2}$ are present in the interstellar medium, but not in amounts that are significant enough to explain the depletion of oxygen. Whittet [62] has examined the oxygen depletion problem in some detail and concludes that organic refractory compounds may explain the extraordinary consumption of oxygen. Another possible solution might be that outside of the dense clouds $\mathrm{H}_{2} \mathrm{O}$ ice may still be retained on grains of large diameter $(>1 \mu \mathrm{m})$ that are opaque to infrared radiation and hence would not exhibit the $3.6 \mu \mathrm{m}$ absorption feature.

After having read $\S 6.1$, one might question whether or not the determinations of the abundance of $\mathrm{O}$ in the gas phase could be wrong because the adopted $f$-value ${ }^{2}$ of the weak line at $1356 \AA$ was in error, much like the indications that we had with the $2325 \AA$ line of C II. A large fraction of the oxygen measurements were made with this intersystem line. Figure 8 shows both the $1356 \AA$ absorption and that from the permitted line at $1302 \AA$ for two sight lines where high velocity features do not compromise our ability to see the damping wings of the strong line. A preliminary comparison of the two by the author indicates that the outcomes for the two lines show exceptionally good agreement with each other.

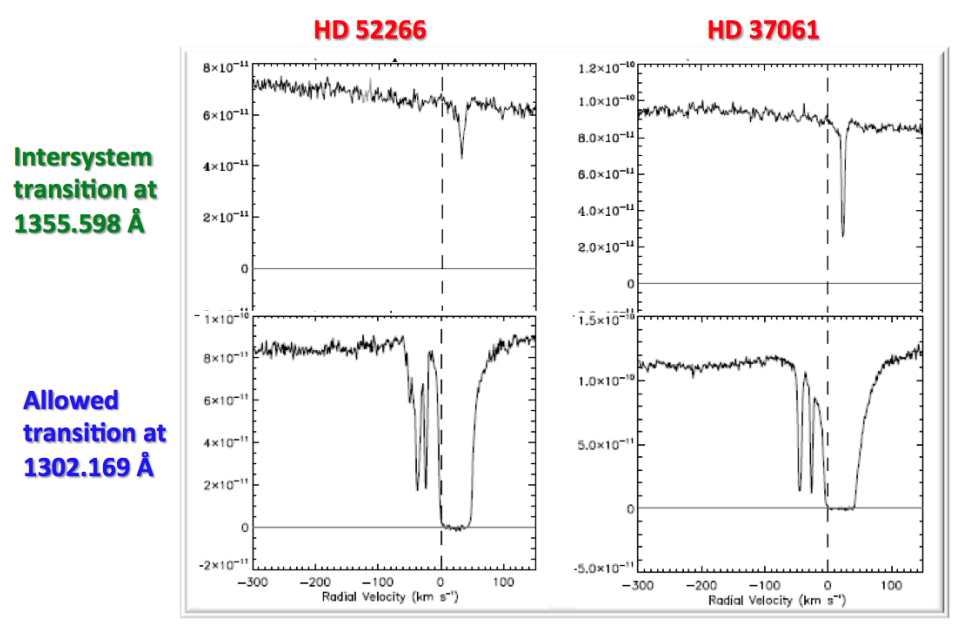

Figure 8: Recordings of spectral segments made by STIS on HST for two stars (labeled at the top). The strengths of the absorptions by the very weak transition at $1356 \AA$ (top panels) are in agreement with the damping wings of the very strongly saturated line at $1302 \AA$ (bottom panels).

\footnotetext{
${ }^{2}$ Jenkins [34] corrected all of the O I determinations based on the $1356 \AA$ line so that they were consistent with an $f$-value equal to $1.16 \times 10^{-6}$ given in the compilation of Morton [63].
} 
An entirely different way to sense the amount of oxygen in bound form is to examine the detailed structure of the K-edge X-ray absorption feature at a wavelength of $23 \AA$. When the oxygen atoms are chemically bound to other atoms, the energies of their electron states are perturbed slightly. The analysis of the absorption structures in the vicinity of the K-edge is rather complicated, owing to the presence of many additional features from higher ionization levels of free oxygen atoms [64]. Pinto, et al. [65] examined the X-ray spectra of nine Galactic low-mass Xray binaries obtained from the Reflection Grating Spectrometer on XMM-Newton. They found that the percentage of oxygen in chemically bound form ranges between 15 and $25 \%$ of the total amount of oxygen present. However, degeneracies in the signatures of different compounds prevented them from identifying the detailed chemical structure of oxygen-bearing substances. Their reported range for the percentage of bound oxygen corresponds to the UV absorption line results for $0.26<F_{*}<0.51$.

\subsection{Krypton}

A surprising result to emerge from the study of krypton abundances is that $\left[\mathrm{Kr}_{\text {gas }} / \mathrm{H}\right]$ varies from an extreme of -0.5 to -0.1 (see the right-most panel in Fig. 2.3). In the latest of a series of investigations of $\mathrm{Kr}$, Cartledge, et al. [66] dismissed the notion that $\mathrm{Kr}$ could be depleted onto grains because it is a noble gas. Instead, they viewed the variations in $\mathrm{Kr}$ abundances as a consequence of differing contributions from nucleosynthetic activity in different regions of space. While this could remain a possibility, it is nevertheless evident that there is a trend that relates $\left[\mathrm{Kr}_{\text {gas }} / \mathrm{H}\right]$ to $F_{*}$, but only at an $89 \%$ level of confidence based on a Pearson correlation coefficient (with a one-tail test because we can reject positive values of $A_{\mathrm{Kr}}$; also note from Table 1 that while $A_{\mathrm{Kr}}$ is negative at only the $1.6 \sigma$ level of significance, ${ }^{3} B_{\mathrm{Kr}}$ is negative at the $4 \sigma$ level). No trend was evident when Cartledge, et al. [66] plotted $\left[\mathrm{Kr}_{\text {gas }} / \mathrm{H}\right]$ against $\left\langle n_{\mathrm{H}}\right\rangle$, which indicates that the average density along a sight line is probably a less accurate indicator of depletion than $F_{*}$, which relies on elements with highly significant depletions as indicators.

It may be possible to understand the krypton depletions in terms of it binding either in the form of a clathrate hydrate [36] or in a complex with $\mathrm{H}_{3}^{+}$[67]. For the former, recall that in $\S 6.3$ we considered the possibility that large quantities of water ices may be on the surfaces of grains with large diameters. For the latter, we know that cosmic ray ionization can result in the creation of fractional abundances of $\mathrm{H}_{3}^{+} / \mathrm{H}_{2}$ as high as $10^{-7}$ in low density regions of the interstellar medium [68]. For either of these means for sequestering $\mathrm{Kr}$, it would be useful to perform calculations of the equilibrium concentrations that might be expected.

\section{Concluding Remarks}

A study of the depletions of different elements from the gas phase of the interstellar medium sets some important constraints on the possible compositions of dust grains in different stages of development or levels of concentration. However, we do not obtain explicit information on what compounds are formed. One can, however, make some illustrative guesses on the possible mixes

\footnotetext{
${ }^{3}$ A. M. Ritchey (private communication) has remeasured many $\mathrm{Kr}$ abundances, and his new results strengthen the significance of the negative slope for $A_{\mathrm{Kr}}$.
} 
of minerals that satisfy the constraints, as had been done by Draine [69], who examined the results from a contemporaneous study of depletions by Jenkins [70].

Some words of caution are in order. There may be some dangers in over-generalizing the results presented in $\S \S 2-3$ when one considers grain compositions in systems other than our immediate neighborhood in our Galaxy. One might suppose that in a system where the proportions of different elements are not the same as what we see here, it would be a simple matter to estimate how rapidly the elements disappear into solid form by just integrating the differential depletion relationships, as expressed in Eq. 3.1, after starting with some initial gas composition. Complications arise when we recognize that some compounds rely on the presence of others to form initially and remain stable for long periods of time. For instance, Lodders [36] has pointed out that the incorporation of the elements $\mathrm{Ni}$ and $\mathrm{Ge}$ in solids depends on the presence of a host element Fe to create an alloy. Likewise, the formation of refractory compounds that contain $\mathrm{Zn}$ and $\mathrm{Mn}$, such as $\mathrm{Zn}_{2} \mathrm{SiO}_{4}$, $\mathrm{ZnSiO}_{3}$, or $\mathrm{Mn}_{2} \mathrm{SiO}_{4}$, are aided by pre-existing host minerals such as forsterite and enstatite. In some galaxy environment where the $\alpha /$ Fe differs from that of our own, some elements may have more or less than their respective host compounds that contain other key elements, thus altering the depletion rates in a way that is difficult to predict. We need also to recognize that any changes in the mix of sources that create the initial grains, such as different kinds of stars and supernovae, can have a profound influence on the outcome. These initial grains are important constituents by themselves, and moreover they eventually form the cores of grains that undergo further development in the interstellar medium.

\section{Bibliography}

[1] L. Spitzer and F.R. Zabriskie, Interstellar research with a spectroscopic satellite, PASP, 71, 412 (1959).

[2] L. Spitzer, Upper Limits on the Abundances of Interstellar Li and Be, ApJ, 109, 548 (1949).

[3] G.H. Herbig, The interstellar line spectrum of Zeta Ophiuchi, Zeitschrift fur Astrophysik, 68, 243 (1968).

[4] A.M. Boesgaard, Interstellar beryllium, PASP, 97, 37 (1985).

[5] G. Wallerstein and D. Goldsmith, The interstellar abundance of titanium, ApJ, 187, 237 (1974).

[6] G.M. Stokes and L.M. Hobbs, Observations of interstellar titanium toward 47 stars, ApJ (Letters), 208, L95 (1976).

[7] G.M. Stokes, Interstellar titanium, Astrophysical Journal Supplements, 36, 115 (1978).

[8] G. Wallerstein and K.K. Gilroy, CCD Observations of Additional Interstellar Lines in Stars Associated with the Vela Remnant and Eta Carinae Nebulosity, Astron. J., 103, 1346 (1992).

[9] B.Y. Welsh, et al., A Minisurvey of Interstellar Titanium from the Southern Hemisphere, Astrophysical Journal Supplements, 112, 507 (1997).

[10] J.X. Prochaska, et al., Evidence for correlated titanium and deuterium depletion in the Galactic ISM, ApJ (Letters), 620, L39 (2005).

[11] S.L. Ellison, et al., The Galactic deuterium abundance and dust depletion: Insights from an expanded Ti/H sample, MNRAS, 380, 1245 (2007). 
[12] D.E. Welty and P.A. Crowther, Interstellar Ti II in the Milky Way and Magellanic Clouds, MNRAS, 404, 1321 (2010).

[13] P.M. Routly and L. Spitzer, A comparison of the components in interstellar sodium and calcium, ApJ, 115, 227 (1952).

[14] R.S. Siluk and J. Silk, On the velocity dependence of the interstellar Na I/Ca II ratio, ApJ, 192, 51 (1974).

[15] J.V. Vallerga, et al., High-Resolution Ca II Observations of the Local Interstellar Medium, ApJ, 411, 729 (1993).

[16] K.R. Sembach and A.C. Danks, Optical studies of interstellar material in low density regions of the Galaxy .2. Cloud properties, kinematics, and distribution of the neutral gas, A\&A, 289, 539 (1994).

[17] J.B. Rogerson, et al., Spectrophotometric Results from the Copernicus satellite. I. Instrumentation and Performance, ApJ (Letters), 181, L97 (1973).

[18] L. Spitzer and E.B. Jenkins, Ultraviolet Studies of the Interstellar Gas, ARAA, 13, 133 (1975).

[19] L.L. Cowie and A. Songaila, High-resolution optical and ultraviolet absorption-line studies of interstellar gas, ARAA, 24, 499 (1986).

[20] D.C. Morton, et al., Spectrophotometric results from the Copernicus satellite. II. Composition of interstellar clouds, ApJ (Letters), 181, L103 (1973).

[21] G.B. Field, Interstellar abundances: gas and dust, ApJ, 187, 453 (1974).

[22] E.B. Jenkins, et al., Abundances of interstellar atoms from ultraviolet absorption lines, ApJ, 301, 355 (1986).

[23] J.C. Brandt, et al., The Goddard High Resolution Spectrograph: Instrument, goals, and science results, Publications of the Astronomical Society of the Pacific, 106, 890 (1994).

[24] J.A. Cardelli, et al., First results from the Goddard High-Resolution Spectrograph: Elemental abundances in the diffuse clouds toward xi Persei, ApJ (Letters), 377, L57 (1991).

[25] J.A. Cardelli, et al., Interstellar Gas Phase Abundance of Carbon, Oxygen, Nitrogen, Copper, Gallium, Germanium, and Krypton Toward zeta Ophiuchi, ApJ (Letters), 383, L23 (1991).

[26] B.D. Savage, et al., First results from the Goddard High-Resolution Spectrograph: Element abundances as a function of velocity in the neutral gas toward xi Persei, ApJ (Letters), 377, L53 (1991).

[27] B.D. Savage, et al., Ultraviolet Observations of the Gas Phase Abundances in the Diffuse Clouds Toward zeta-Ophiuchi at $3.5 \mathrm{Km} / \mathrm{s}$ Resolution, ApJ, 401, 706 (1992).

[28] J.A. Cardelli, et al., Ultraviolet Transitions of Low Condensation Temperature Heavy Elements and New Data for Interstellar Arsenic, Selenium, Tellurium, and Lead, ApJ (Letters), 416, L41 (1993).

[29] U.J. Sofia, et al., High-Resolution Ultraviolet Observations of the Interstellar Diffuse Clouds Toward ти Columbae, ApJ, 413, 251 (1993).

[30] J.A. Cardelli, The abundance of heavy elements in interstellar gas, Science, 265, 209 (1994).

[31] B.D. Savage and K.R. Sembach, Interstellar abundances from absorption line observations with the Hubble Space Telescope, ARAA, 34, 279 (1996).

[32] B.E. Woodgate and et al., The Space Telescope Imaging Spectrograph (STIS) design, PASP, 110, 1183 (1998). 
[33] H.W. Moos, et al., Overview of the Far Ultraviolet Spectroscopic Explorer Mission, ApJ, 538, L1 (2000).

[34] E.B. Jenkins, A unified representation of gas-phase element depletions in the interstellar medium, ApJ, 700, 1299 (2009).

[35] T.P. Snow, et al., Gas-Phase Iron Abundances and Depletions in Translucent Interstellar Lines of Sight from Far Ultraviolet Spectroscopic Explorer Observations of Fe II Lines, ApJ, 573, 662 (2002).

[36] K. Lodders, Solar system abundances and condensation temperatures of the elements, ApJ, 591, 1220 (2003).

[37] M.F. Nieva and N. Przybilla, Present-day cosmic abundances - A comprehensive study of nearby early B-type stars and implications for stellar and Galactic evolution, and interstellar dust models, A\&A, 539, 143N (2012).

[38] M. Asplund, et al., The chemical composition of the Sun, ARAA, 47, 481 (2009).

[39] L.S. Lyubimkov, Possible Overionization of C II, N II, and O II Ions in the Atmospheres of Early B- and Late O-Type Stars, Astrophysics, 56, 472 (2013).

[40] T.P. Snow, The depletion of interstellar elements and the interaction between gas and dust in space, ApJ (Letters), 202, L87 (1975).

[41] A. Klotz, et al., Contribution of metal-bonded polycyclic aromatic hydrocarbons to the interstellar depletion, A\&A, 304, 520 (1995).

[42] M.J. Barlow, The destruction and growth of dust grains in interstellar space - I. Destruction by sputtering, MNRAS, 183, 367 (1978).

[43] I. Ramírez, et al., A possible signature of terrestrial planet formation in the chemical composition of solar analogs, A\&A, 521, 33 (2010).

[44] I. Ramírez, et al., Accurate abundance patterns of solar twins and analogs. Does the anomalous solar chemical composition come from planet formation?, A\&A, 508, L17 (2009).

[45] J. Meléndez, et al., The Peculiar Solar Composition and Its Possible Relation to Planet Formation, ApJ (Letters), 704, L66 (2009).

[46] I. Ramírez, et al., Chemical signatures of planets: beyond solar-twins, A\&A, 561, 7 (2014).

[47] T.P. Snow and A.N. Witt, The interstellar carbon budget and the role of carbon in dust and large molecules, Science, 270, 1455 (1995).

[48] J.S. Mathis, Dust models with tight abundance constraints, ApJ, 472, 643 (1996).

[49] S.H. Kim and P.G. Martin, On the dust-to-gas ratio and large particles in the interstellar medium, ApJ, 462, 296 (1996).

[50] J.A. Cardelli, et al., The abundance of interstellar carbon, ApJ, 467, 334 (1996).

[51] E. Dwek, Can Composite Fluffy Dust Particles Solve the Interstellar Carbon Crisis?, ApJ, 484, 779 (1997).

[52] A. Li, Can fluffy dust alleviate the subsolar interstellar abundance problem?, ApJ, 622, 965 (2005).

[53] B.T. Draine and H.M. Lee, Optical properties of interstellar graphite and silicate grains, ApJ, 285, 89 (1984).

[54] U.J. Sofia, et al., Determining interstellar carbon abundances from strong-line transitions, Astron. J., 141, 22 (2011). 
[55] H.P. Gail and E. Sedlmayr, The primary condensation process for dust around late M-type stars, A\&A, 166, 225 (1986).

[56] D.C. Knauth, et al., Discovery of interstellar N2 with the Far Ultraviolet Spectroscopic Explorer, Nature, 429, 636 (2004).

[57] —, Discovery of interstellar N2, in Astrophysics in the Far Ultraviolet, Five Years of Discovery with FUSE, G. Sonneborn, H. W. Moos and B. G. Andersson, eds., (San Francisco, Ast. Soc. Pacific), p. 421 (2006).

[58] E.F. van Dishoeck, ISO spectroscopy of gas and dust: From molecular clouds to protoplanetary disks, ARAA, 42, 119 (2004).

[59] D.C.B. Whittet, et al., Infrared spectroscopy of dust in the Taurus dark clouds: ice and silicates, MNRAS, 233, 321 (1988).

[60] C. Eiroa and K.W. Hodapp, Ice dust grains in the Serpens molecular cloud, A\&A, 210, 345 (1989).

[61] R.G. Smith, et al., Grain mantles in the Taurus dark cloud, MNRAS, 263, 749 (1993).

[62] D.C.B. Whittet, Oxygen depletion in the interstellar medium: Implications for grain models and the distribution of elemental oxygen, ApJ, 710, 1009 (2010).

[63] D.C. Morton, Atomic data for resonance absorption lines. III. Wavelengths longward of the Lyman limit for the elements hydrogen to gallium, Astrophysical Journal Supplements, 149, 205 (2003).

[64] A.M. Juett, et al., High-resolution x-ray spectroscopy of the interstellar medium: structure at the oxygen absorption edge, ApJ, 612, 308 (2004).

[65] C. Pinto, et al., ISM composition through X-ray spectroscopy of LMXBs, A\&A, 551, A25 (2013).

[66] S.I.B. Cartledge, et al., Interstellar krypton abundances: The detection of kiloparsec-scale differences in Galactic nucleosynthetic history, ApJ, 687, 1043 (2008).

[67] O. Mousis, et al., Sequestration of noble gases by H3+ in protoplanetary disks and outer solar system composition, ApJ, 673, 637 (2008).

[68] N. Indriolo and B.J. McCall, Investigating the cosmic-ray ionization rate in the Galactic diffuse interstellar medium through observatons of H3+, ApJ, 745, 91 (2012).

[69] B.T. Draine, Interstellar Dust, in Origin and Evolution of the Elements, A. Mc William and M. Rauch, eds., (Cambridge, Cambridge Univ. Press), p. 317 (2004).

[70] E.B. Jenkins, Interstellar Atomic Abundances, in Origin and Evolution of the Elements, A. Mc William and W. Rauch, eds., (Cambridge, Cambridge Univ. Press), p. 336 (2004). 